\title{
Metodologías cooperativas y colaborativas en la formación del profesorado para la interculturalidad
}

Cooperative and collaborative methodologies in teacher training for interculturality.

\author{
Alicia Peñalva Vélez \\ e-mail: alicia.penalva@unavarra.es \\ Universidad Pública de Navarra. España \\ Juan José Leiva Olivencia (iD) \\ e-mail: juanleiva@uma.es \\ Universidad de Málaga. España
}

\section{Resumen}

La sociedad red actual y el sistema educativo del siglo XXI demandan una formación específica para el profesorado, centrada en la capacitación del mismo para la atención a la diversidad cultural e identitaria. En el ámbito educativo se entiende que el modelo intercultural es el más adecuado para gestionar la diversidad, y formar a la ciudadanía crítica. Los objetivos de este estudio son definir qué son las metodologías activas (cooperativa y colaborativa), y definir su función en el marco de la formación del profesorado para la interculturalidad. De esta forma se podrá garantizar que la formación sea: de tipo técnico-pedagógica, de tipo procedimental y/o metodológica, centrada en el aprendizaje activo del alumnado, y en un enfoque de la enseñanza basado en el aprendizaje por competencias. Todos ellos aspectos básicos para la formación del profesorado para la interculturalidad.

Palabras clave: Formación del profesorado; interculturalidad; metodologías activas.

\begin{abstract}
Current network society and inclusive schools needs an specific training for teachers, focused intercultural education and in cultural diversity and identity. In the educational field, it is understood that the intercultural model is the most adequate to manage diversity and train critical citizenship. The objectives of this study are to define what active methodologies are (cooperative and collaborative), and to define their function within the framework of teacher training for interculturality. In this way it will be possible to guarantee that the training is: of a technical-pedagogical type, of procedural and / or methodological type, focused on the active learning of the students, and on a teaching approach based on competency-based learning. All of them are basic aspects for teacher training for interculturality.
\end{abstract}

Keywords: Teacher training; interculturality; active methodologies.

Recibido / Received: 09-03-2018

Aceptado / Accepted: 04-10-2018

Cómo referenciar este artículo / How to reference this article:

Peñalva Vélez, A., \& Leiva Olivencia, J.J. (2019). Metodologías cooperativas y colaborativas en la formación del profesorado para la interculturalidad. Tendencias Pedagógicas, 33, 37-46. doi: $10.15366 /$ tp2019.33.003 


\section{Introducción}

La sociedad red actual (Castells, 2006) y el sistema educativo del siglo XXI (Peñalva-Vélez, López-Goñi y Barrientos, 2017) demandan una formación específica para el profesorado, centrada en la capacitación del mismo para la atención a la diversidad cultural e identitaria (Peñalva Vélez y López-Goñi, 2014; Ortega-Velasco, 2011; Leiva y Escarbajal, 2011). Dicha formación se viene abordando en el plano educativo según Leiva (2010) desde planteamientos interculturales. El mismo autor señala que en el ámbito educativo se entiende que el modelo intercultural es el más adecuado para gestionar la diversidad, y formar a la ciudadanía crítica (Aguado, 2005; Leiva, 2011). Se puede afirmar por lo tanto que la potencialidad de implementar una educación intercultural e inclusiva ha pasado de ser un propósito de determinados países y sistemas educativos, a convertirse en un objetivo absolutamente clave de la agenda política mundial.

En este sentido, como señalan Peñalva-Vélez y López-Goñi (2014) la formación del profesorado para la interculturalidad debería desarrollar la competencia intercultural como una combinación de capacidades específicas: (1) actitudes positivas hacia la diversidad cultural; (2) capacidad comunicativa; (3) capacidad de manejar conflictos en situaciones interculturales; y (4) capacidad de tener conciencia sobre la propia cultura y sobre cómo ésta influye en la visión e interpretación de la realidad (Jandt, 1995; Deardorff, 2009). Sin embargo la realidad muestra que en el ámbito de la educación superior los docentes no están siendo formados para atender las necesidades de la realidad intercultural, algo que atenta directamente contra un factor básico de la calidad educativa (Peñalva-Vélez y Leiva, en prensa; Soriano, 2005). Se hace caso omiso como señalan Palomero (2006) y Trujillo (2010) a una de las inquietudes que presenta el profesorado: la formación específica en interculturalidad.

En este sentido también Santos-Rego, Cernadas-Ríos y Lorenzo-Moledo (2014) indican la evidencia de que en general el profesorado no ha recibido, ni en su formación inicial ni en la permanente, una preparación específica para la elaboración de verdaderos currículos interculturales. Aún a pesar de que, como indican distintos autores, la educación intercultural se va a poder desarrollar correctamente si y sólo si el profesorado adquiere aquellas competencias interculturales que les son necesarias para desenvolverse eficazmente en un medio intercultural (Leiva, 2010; Santos-Rego et al., 2014). En este sentido Essomba (2015) remarca que la formación de profesionales de la educación en diversidad cultural e interculturalidad todavía dispone de recorrido, a pesar de la evidencia de que en general el profesorado no ha recibido ni en su formación inicial ni en la permanente, una preparación específica para elaborar verdaderos currículos interculturales (Santos-Rego, Cernadas-Ríos y Lorenzo-Moledo, 2014). Nuevamente Essomba (2015) señala que "debemos invertir esfuerzos colectivos para incrementar la calidad de las propuestas y, en consecuencia, la efectividad de los impactos transformativos en la realidad educativa" (p.128).

A la hora de abordar el diseño de un plan de formación en el que se establezcan las líneas pedagógicas, didácticas y curriculares básicas en relación a la interculturalidad, se deben tener en cuenta cuatro aspectos o ámbitos de formación docente: (1) los contenidos interculturales, para conocer qué son y qué significan las otras culturas, (2) la capacidad de análisis crítico de las informaciones que se transmiten sobre los "otros" diferentes, (3) el desarrollo de habilidades para entender y respetar otros modos de percibir la realidad, (4) la capacidad de valorar otros culturas e identidades culturales, para fomentar y promover el contacto entre ellas (Peñalva Vélez y Aguilar, 2011). Esta formación de tipo inicial debe ser: (a) instrumental -para facilitar una idea abierta y compleja de la interculturalidad-, (b) conceptual -en torno al conocimiento propio de la interculturalidad, y las habilidades cognitivas y sociales que implica su desarrollo práctico-, (c) autorreflexiva -con la diversidad cultural como eje definitorio y transversal del quehacer docente-, (d) crítico-situacional -para la creación de criterios profesionales fundamentados que guíen las actuaciones educativas interculturales-, y (e) técnico-pedagógica -que permita una aproximación amplia a las posibilidades didácticas que ofrece la interculturalidad desde el punto de vista del curriculum (Leiva, 2012).

Por último, en la formación intercultural del profesorado deberían plantearse las siguientes 
dimensiones formativas: la cognitiva (referida a la necesidad de conocer las culturas de los alumnos inmigrantes presentes en nuestros centros educativos), la actitudinal (referida a la receptividad que muestra el profesorado antes la diversidad cultural que representa la presencia de alumnado de origen inmigrante), la ética (referida a la predisposición moral con la que el profesorado concibe la diversidad en el mundo y en la escuela), la emocional (que plantea la necesidad de estudiar las identidades individuales a través del reconocimiento de la existencia de identidades culturales plurales), la procedimental y/o metodológica (que es la que tiene que ver con todo el conjunto de habilidades, destrezas y capacidades de tipo práctico para llevar los principios de la educación intercultural a las aulas y escuelas), la de mediación (que parte de la valoración positiva de la mediación intercultural en la mejora de la convivencia en los centros educativos) (Leiva, 2010, 2011 y 2012).

En la tabla 1 se recogen los aspectos básicos que se deben concretar a la hora de plantear una formación inicial y permanente del profesorado en los principios y presupuestos del paradigma intercultural. Se indican en primer lugar las cuatro capacidades específicas que se deben desarrollar para la consecución de la competencia intercultural. Se continúa indicando en qué temáticas específicas debe centrase la formación. Y se concluye indicando cómo debe ser la formación en sí misma, y qué dimensiones formativas debe plantear.

Tabla 1

Aspectos básicos de la formación del profesorado para la interculturalidad

\begin{tabular}{ll}
\hline Aspectos básicos & Concreción de cada aspecto \\
\hline (I) Debe tener como & (1) actitudes positivas hacia la diversidad cultural \\
objetivo el desarrollo de la & (2) capacidad comunicativa \\
competencia intercultural & (3) capacidad de manejar conflictos en situaciones interculturales \\
como combinación de 4 & (4) capacidad de tener conciencia sobre la propia cultura y sobre \\
capacidades específicas: & cómo ésta influye en la visión e interpretación de la realidad \\
& (1) los contenidos interculturales \\
& (2) la capacidad de análisis crítico \\
(II) Se debe centrar en: & (3) el desarrollo de habilidades para entender y respetar otros modos \\
& de percibir la realidad \\
& (4) la capacidad de valorar otras culturas e identidades culturales \\
& (1) instrumental \\
(III) Debe ser: & (2) conceptual \\
& (3) autorreflexiva \\
& (4) crítico-situacional \\
(IV) Debe plantearse las & (5) técnico-pedagógica \\
siguientes dimensiones & (1) la cognitiva \\
formativas: & (2) la actitudinal \\
& (4) la ética
\end{tabular}

Fuente: elaboración propia

Dado que el fin principal es lograr, bien a través de la formación inicial, bien a través de la formación permanente, la competencia intercultural en el profesorado, resulta fundamental trabajar desde la perspectiva de las metodologías activas, basado en el enfoque de Fernández-March (2006, p.4): el del aprendizaje a lo largo de la vida. Algo que implica necesariamente un cambio en la concepción metodológica tradicional, y la adopción de un enfoque de trabajo a través de metodologías activas. Por eso la finalidad de este estudio es presentar los principales rasgos definitorios de las metodologías cooperativa y colaborativa. Para poder llevarlo a cabo se va a proceder a revisar ambos conceptos de cara a presentar en forma de compendio, los rasgos que de 
manera principal los identifican y diferencian entre sí. Resulta difícil encontrar un compendio como el que ahora se presenta en ningún documento que no sea un libro o un capítulo de libro. Por todo ello se puede afirmar que los objetivos de este estudio son definir qué son las metodologías activas (cooperativa y colaborativa), y definir su función en el marco de la formación del profesorado para la interculturalidad.

De esta forma se podrá garantizar que la formación sea de tipo técnico-pedagógica y permita al profesorado una aproximación amplia a las posibilidades didácticas que ofrece la interculturalidad desde el punto de vista del curriculum (Tabla 1, aspecto III -5-). Permitirá así mismo plantear la dimensión formativa de tipo procedimental y/o metodológica, y abordar por lo tanto el conjunto de habilidades, destrezas y capacidades de tipo práctico necesarios para llevar los principios de la educación intercultural a las aulas y escuelas (Tabla 1, aspecto IV -5-). Y permitirá sobre todo plantear la formación para la interculturalidad del profesorado, y el futuro trabajo en las aulas de dicho profesorado, desde un modelo educativo centrado en el aprendizaje activo del alumnado. Centrado en un enfoque de la enseñanza basado en el aprendizaje por competencias, desde la perspectiva del aprendizaje situado, significativo y funcional (Tabla 1, aspecto I).

\section{Aprendizaje cooperativo y aprendizaje colaborativo}

Actualmente, metodológicamente hablando, las instituciones educativas han vivido un cambio de concepción y metodología, que les ha ayudado a caminar desde enfoques educativos más tradicionales hacia enfoques basados en el aprendizaje por competencias (Rubia y Guitert, 2014). Los procesos de formación se vuelven más abiertos, grupales, sociales y dinámicos. En ello influyen teorías sobre el aprendizaje de autores como Piaget o Vigotsky desde el plano más psicológico, y Freinet o Dewey desde un plano más pedagógico. Tal y como sostiene Fernández-March (2006, p.36): "Asistimos a la transición de un modelo educativo centrado en la enseñanza hacia un modelo centrado en el aprendizaje". Los rasgos principales de este nuevo modelo educativo son que se centra en el aprendizaje, que exige el giro del "enseñar" al "aprender" y principalmente enseñar a aprender a aprender y aprender a lo largo de la vida. Se centra además en el aprendizaje autónomo del estudiante tutorizado por los profesores, y en los resultados de aprendizaje, expresadas en términos de competencias genéricas y específicas. El cambio de modelo educativo se refuerza con el enfoque de la enseñanza basado en competencias, y el aprendizaje por competencias se centra de manea básica en la significatividad y la funcionalidad de los aprendizajes, a partir de un aprendizaje situado.

En este contexto adquieren todo su sentido las que conocemos como "metodologías activas", un concepto que como señalan Labrador y Andreu (2008) no es nuevo. A finales del siglo XIX y principios del siglo XX se inicia el movimiento de renovación educativa y pedagógica de la Escuela Nueva o Escuela Activa, basado en una serie de principios que derivan en una nueva comprensión de las necesidades de la infancia. González-Fernández y García-González (2012) señalan que la participación activa de todos los integrantes educativos en los procesos de educación, en la línea que marcan los principios de la Escuela nueva conduce a la puesta en marcha de cinco modalidades de aprendizaje:

- El aprendizaje activo: cada persona tiene que aprender por sí misma.

- El aprendizaje autorregulado: cada estudiante debe percibir sus propias actividades correctamente, evaluar los resultados de las mismas.

- El aprendizaje constructivo: el conocimiento individual no es una copia de la realidad, al menos en parte, es una construcción personal.

- El aprendizaje situado: el aprendizaje es un proceso situado cuando el contexto de aprendizaje ofrece oportunidades de aplicar los conocimientos adquiridos.

- El aprendizaje social: aprender es un proceso social, cada proceso de enseñanza-aprendizaje es una interacción social.

Como indican Labrador y Andreu (2008) "por metodologías activas se entiende hoy en día aquellos métodos, técnicas y estrategias que utiliza el docente para convertir el proceso de 
enseñanza en actividades que fomenten la participación activa del estudiante y llevan al aprendizaje" (p.6). Fomentan la participación activa del alumnado en su proceso educativo y favorecen el aprendizaje significativo. Los componentes básicos a tener en cuenta a la hora de diseñar estos métodos docentes son la organización de los espacios, la selección del método adecuado a cada situación de enseñanza, la orientación y gestión de las actividades de aprendizaje, y las relaciones interpersonales que se establecen durante las actividades (Fernández-March, 2006). Igualmente, los nuevos entornos TIC generan nuevas formas de enseñar los docentes y aprender los estudiantes, que se basan en orientaciones, espacios, procedimientos y recursos diferenciales (Páramo, Pérez y Ruiz, 2016).

También las metodologías activas forman parte indispensable de estos nuevos entornos, a los que proporcionan nuevos procesos, procedimientos, técnicas y herramientas que implican de manera activa al estudiante en su proceso de aprendizaje mediado por dispositivos tecnológicos (Pérez-Tornero y Tejedor, 2016). La implementación de metodologías mediante el uso de las TIC remite directamente al e-learning, que permite plantear el aprendizaje flexible, interactivo y centrado en el estudiante, y, ante todo, apoyado en las tecnologías. Un aprendizaje que se produce por lo tanto en diferentes espacios y contextos mediados por TIC, que no se ata a un espacio único, y que se ajusta a las particularidades del que enseña y aprende. Engloba, entre otros el B-learning y el Mlearning (Páramo, Pérez y Ruiz, 2016, p.85).

¿Qué papel juegan el aprendizaje cooperativo y colaborativo en este cambio de concepción educativa y metodológica descrito? Todo proceso de enseñanza-aprendizaje implica una planificación y una toma de decisiones respeto a cómo se estructure el espacio y se planteen las actividades. Según Sánchez-Queija y Pertegal (2014) la estructura de funcionamiento en el aula puede ser de tres tipos básicamente: individual, competitiva o cooperativa. Como explica Pujolàs (2012) la estructura cooperativa supone que la forma habitual de trabajar en el grupo-clase sea realizar las actividades y tareas en grupo. La colaboración y cooperación entre niños y niñas es la fórmula ideal para aprender. Esta forma cooperativa de aprender, que se produce en grupo e incluso en pareja, es un aprendizaje entre iguales que incluye tres formas diferentes de aprender conjuntamente: tutoría entre iguales, aprendizaje cooperativo y aprendizaje colaborativo (SánchezQueija y Pertegal, 2014).

Según indican Barkley, Cross y Major (2012) para la mayoría de los educadores los términos colaborativo y cooperativo tienen significados similares. Unos autores utilizan los términos cooperativo y colaborativo de manera intercambiable, otros hacen una clara distinción epistemológica entre ellos, y señalan que el aprendizaje cooperativo difiere del colaborativo en que, en el primero, la utilización de grupos apoya un sistema de enseñanza que mantiene las líneas tradicionales del saber y la autoridad en el aula. Para otros autores, el aprendizaje cooperativo no es más que una subcategoría del colaborativo. El aprendizaje colaborativo, al igual que la tutoría y el aprendizaje cooperativo, son tres formas diferentes de aprender conjuntamente entre iguales. La diferencia entre estas tres formas se basa en dos dimensiones: la mutualidad (o grado de implicación en la comunicación entre los participantes) y la igualdad (o igualdad de roles entre los participantes). Tal y como indican Prados et al. (2014, p.141), gráficamente se puede explicar como sigue (Tabla 2):

Tabla 2

Tipos de aprendizaje en función de la mutualidad y la igualdad

\begin{tabular}{lll}
\hline $\begin{array}{l}\text { Tipo de } \\
\text { aprendizaje }\end{array}$ & Igualdad & Mutualidad \\
\hline Tutoría & Baja & $\begin{array}{l}\text { Depende de la habilidad del tutor y de la implicación del tutorizado } \\
\text { Depende del grado en que se anime el aprendizaje grupal y de las recompensas } \\
\text { por trabajar en grupo (motivación intrínseca o extrínseca) }\end{array}$ \\
$\begin{array}{lll}\text { Cooperativo } \\
\text { Colaborativo }\end{array}$ & Alta & Alta
\end{tabular}

Fuente: elaboración propia a partir de Prados et al., 2014, p.141.

Lo cierto es que como señalan Barkley, Cross y Major (2012), resulta necesario distinguir el 
aprendizaje cooperativo y colaborativo puesto que cada uno tiene unas características que se pueden considerar esenciales y exclusivas, ya que además los fundamentos epistemológicos de ambos son diferentes. La definición más directa del aprendizaje cooperativo es el uso de la enseñanza de pequeños grupos para que los alumnos trabajen juntos con el fin de maximizar el aprendizaje. El aprendizaje cooperativo surgió principalmente como una alternativa a lo que parecía una insistencia excesiva de la educación tradicional en la competición. El aprendizaje colaborativo se basa en supuestos epistemológicos diferentes y tiene su origen en el constructivismo social. El aprendizaje colaborativo se produce cuando los alumnos y los profesores trabajan juntos para crear el saber. En su definición más estricta, parte de la base de que el saber se produce socialmente por consenso entre compañeros versados en la cuestión.

En definitiva y de manera general, quienes optan por distinguir entre aprendizaje cooperativo y colaborativo señalan que la diferencia entre ellos es que mientras la educación cooperativa parece ser más apropiada para los niños, el aprendizaje colaborativo es más adecuado para los estudiantes universitarios. Zarzar (2016) indica que aprendizaje cooperativo y colaborativo aun siendo semejantes como ya se ha dicho, implican planteamientos diferentes. Una de las diferencias fundamentales se localiza en el papel del profesor. Mientras que en el aprendizaje cooperativo es quien define el rumbo de trabajo, en el aprendizaje colaborativo el profesor deposita la autoridad en el grupo, que se vuelve autogestionado. El propio Zarzar (2016, p.56), señala de manera gráfica las diferencias entre ambos aprendizajes como sigue (Tabla 3):

Tabla 3

Diferencias entre el aprendizaje cooperativo y colaborativo

\begin{tabular}{|c|c|c|}
\hline Punto de vista & Aprendizaje cooperativo & Aprendizaje colaborativo \\
\hline $\begin{array}{l}\text { Origen filosófico y } \\
\text { epistemológico }\end{array}$ & $\begin{array}{l}\text { Propuesta técnica y pedagógica } \\
\text { que se utiliza como alternativa a } \\
\text { la educación tradicional }\end{array}$ & $\begin{array}{l}\text { Tiene sus orígenes en el } \\
\text { constructivismo social. El } \\
\text { aprendizaje se construye a través } \\
\text { de los procesos sociales en los } \\
\text { que se involucra la persona }\end{array}$ \\
\hline $\begin{array}{l}\text { Relación con el sistema } \\
\text { educativo }\end{array}$ & $\begin{array}{l}\text { Nueva alternativa para trabajar } \\
\text { dentro del sistema educativo }\end{array}$ & $\begin{array}{l}\text { Se cuestiona el statu quo de los } \\
\text { sistemas educativos actuales }\end{array}$ \\
\hline Papel de autoridad del profesor & $\begin{array}{l}\text { El profesor es el centro de la } \\
\text { autoridad de la clase, el que } \\
\text { define el rumbo }\end{array}$ & $\begin{array}{l}\text { El profesor deposita la } \\
\text { autoridad en el grupo. El grupo } \\
\text { es autogestivo }\end{array}$ \\
\hline $\begin{array}{l}\text { Cómo se definen las actividades } \\
\text { a realizar }\end{array}$ & $\begin{array}{l}\text { El profesor define las } \\
\text { actividades a desarrollar y las } \\
\text { tareas a realizar }\end{array}$ & $\begin{array}{l}\text { Las tareas son definidas de } \\
\text { común acuerdo con el grupo }\end{array}$ \\
\hline Tipos de tareas a realizar & $\begin{array}{l}\text { Suelen ser concretas, específicas } \\
\text { y cerradas }\end{array}$ & $\begin{array}{l}\text { Son más abiertas, menos } \\
\text { estructuradas y más complejas }\end{array}$ \\
\hline Uso de técnicas específicas & $\begin{array}{l}\text { Se han creado gran variedad de } \\
\text { técnicas y estrategias } \\
\text { instruccionales como apoyo } \\
\text { para el trabajo docente }\end{array}$ & $\begin{array}{l}\text { No hay variedad de estrategias, } \\
\text { ya que no es papel del profesor } \\
\text { el diseñarlas y aplicarlas }\end{array}$ \\
\hline $\begin{array}{l}\text { Mecanismos y criterios para la } \\
\text { evaluación }\end{array}$ & El profesor los define & $\begin{array}{l}\text { Se definen de manera } \\
\text { compartida con el grupo }\end{array}$ \\
\hline
\end{tabular}

Fuente: elaboración propia a partir de Zarzar, 2016, p.56.

Con todo, es necesario igualmente recordar que para ambos el propósito de la educación es construir comunidades de aprendizaje que tengan como objetivo preparar a los ciudadanos del futuro. Por lo que ambos dan importancia a la participación activa del estudiante en el trabajo en grupos pequeños, frente a la enseñanza pasiva. En ambos los grupos pequeños siempre tienen tareas específicas que realizar. Ambos se fundamentan en una aproximación al aprendizaje basada en el descubrimiento. En ambos a los participantes en los equipos de trabajo se les asignan roles (aunque son menos los roles asignado en el caso del aprendizaje colaborativo). En ambos los estudiantes deben poseer habilidades para el trabajo grupal, aunque en el aprendizaje cooperativo 
esto se plantea explícitamente como un objetivo de aprendizaje. En ambos casos el trabajo grupal se da dentro de un marco previamente establecido, aunque en el aprendizaje cooperativo este marco está más estructurado que en el aprendizaje colaborativo.

\section{La educación intercultural a través de metodologías de tipo cooperativo y colaborativo}

La función que desempeñan las metodologías activas en el marco de la formación del profesorado para la interculturalidad es doble. Por un lado, estas metodologías, por su propia definición y fundamentación teórico-práctica, son las que mejor contribuyen al desarrollo de competencias. Por otro lado, por el hecho de que el profesorado formado a partir de estas metodologías, será capaz de emplearlas de manera activa a la hora de definir y poner en marcha su propia actividad docente, desde los principios de la interculturalidad y la inclusión. Para Riera (2011) la escuela inclusiva es la que asume el compromiso de atender a todos sin excluir a nadie, y de hacerlo además según sus necesidades educativas. Es una escuela que agrupa al alumnado de manera heterogénea, y que se centra en el desarrollo de una estructura de aprendizaje de tipo cooperativo, no individualista ni competitiva. Atiende a la diversidad, se basa en un enfoque constructivista de la enseñanza y el aprendizaje, y tiene como prioridad el aumento de las interacciones de los alumnos, ya que las considera como factores básicos de su aprendizaje individual y grupal. Por ello el aprendizaje cooperativo y colaborativo se consideran metodologías fundamentales en la construcción de esta escuela inclusiva que debe además ser intercultural.

Para Banks (2008) la educación intercultural nutre e impregna los principios de una educación inclusiva, donde el referente pedagógico por excelencia es la vivencia y convivencia de la diferencia cultural y social como factor de enriquecimiento educativo. Aprovechar educativamente la diversidad cultural pasa por reconocer que lo común es la diversidad y la diversidad debe ser, desde la perspectiva pedagógica, lo común en los diseños y propuestas de intervención educativa (López Melero, 2004). Según Santamaría (n.d) (en Malik y Sánchez, 2006, p.204-207) la metodología o estructura de aprendizaje cooperativa contribuye notablemente a la mejora de los aprendizajes, y el aprendizaje cooperativo tiene las siguientes ventajas para los procesos educativos interculturales:

- Responde a las necesidades de una sociedad multicultural

- Contribuye al desarrollo cognitivo, consiguiendo aumentar la variedad y riqueza de experiencias que proporciona la escuela

- Reduce la ansiedad y fomenta la autoestima de los alumnos

- Fomenta la interacción

- Fomenta la autonomía e independencia

- Permite la adecuación de los contenidos al nivel de los alumnos

- Promueve el desarrollo de destrezas complejas de pensamiento crítico

- Favorece el desarrollo socio-afectivo

- Aumenta la motivación hacia el aprendizaje escolar

- Mejora el rendimiento académico

- Contribuye a reducir la violencia en la escuela

Escarbajal (2010, 2011) afirma que si hablamos de educar interculturalmente mediante procedimientos colaborativos enseguida hemos de pensar en los instrumentos que se consideran idóneos para conseguir estas situaciones: las estrategias de trabajo grupal. Las personas no son sólo producto de las situaciones sociales sino creadoras de ellas; son, por tanto, capaces de modificarlas y recrearlas. Ello se hace, fundamentalmente, a través del intercambio de significados, producto de la interacción social experimentada en el desarrollo de estrategias cualitativas, mediante el aprendizaje colaborativo. De este modo, el conocimiento significativo se torna en un tipo de conciencia compartida: todos aprenden de todos, cada uno a su nivel y desde su experiencia, lo que conlleva procesos de transformación personal y de la práctica educativa. El trabajo con estrategias colaborativas no sólo es un procedimiento metodológico recurrente para trabajar la diversidad en el aula, sino que es mucho más, es un elemento educativo imprescindible tanto para los alumnos como para los profesores. 
En este trabajo se debe potenciar lo que se llama inteligencia cultural, aquella que crece con la comunicación, la del aprendizaje dialógico en donde cada miembro del grupo aporta su cultura y experiencia para compartirla con los demás. Otra ventaja del trabajo colaborativo es el reparto de la carga cognitiva y afectiva que supone realizar una tarea que requiere esfuerzo mental; así se afianza la idea de que es posible alcanzar objetivos difíciles con la ayuda de todos, se reparte responsabilidad y esfuerzo, se motiva y se eleva la autoestima. El trabajo colaborativo permite un aprendizaje cara a cara con interacciones directas; con él se alcanza un nivel más profundo y permanente de comprensión, pensamiento crítico y creativo, a la vez que se forman actitudes positivas hacia los demás y mayores niveles de confianza en el conocimiento y capacidades.

\section{Conclusiones}

Como señala Fernández-March (2006) todo proceso de enseñanza busca crear un proceso de aprendizaje en un contexto concreto, en un momento concreto, y en base a unos objetivos concretos. Para ello se necesita disponer de una metodología, que la autora define como "el conjunto de oportunidades y condiciones que se ofrecen a los estudiantes" (p.41). Además deben organizarse de manera sistemática e intencional. Hay que tener en cuenta además que todo método tiene unos fundamentos en base a los que se define: (1) la interrelación que se va a establecer entre el docente y alumnado, (2) cómo se organiza la actividad del alumnado sobre el objeto de estudio, (3) qué proceso de asimilación del contenido de la enseñanza va a seguir el alumnado. En este sentido González-Fernández y García-González (2012) señalan que la participación activa de todos los integrantes educativos en los procesos de educación, en la línea que marcan los principios de la Escuela nueva y del actual enfoque de la enseñanza basada en competencias, conduce a la puesta en marcha de cinco modalidades de aprendizaje:

- El aprendizaje activo: cada persona tiene que aprender por sí misma.

- El aprendizaje autorregulado: cada estudiante debe percibir sus propias actividades correctamente, evaluar los resultados de las mismas.

- El aprendizaje constructivo: el conocimiento individual no es una copia de la realidad, al menos en parte, es una construcción personal.

- El aprendizaje situado: el aprendizaje es un proceso situado cuando el contexto de aprendizaje ofrece oportunidades de aplicar los conocimientos adquiridos.

- El aprendizaje social: aprender es un proceso social, cada proceso de enseñanza-aprendizaje es una interacción social.

Los enfoques teóricos subyacentes a todos ellos son: (1) el constructivismo, con su principios de significatividad y funcionalidad de los aprendizajes; (2) el enfoque sociocultural, que considera que el aprendizaje es un proceso de interacción entre el sujeto y el medio, y lo fundamenta en principios como el de la interacción continua del profesor con los estudiantes y de los estudiantes entre sí; (3) el enfoque dialógico, que percibe el diálogo como indispensable para la construcción del conocimiento. Entiende el diálogo como la acción conjunta del alumnado, de las familias, la comunidad y los profesionales de la educación.

Tanto el aprendizaje cooperativo como el colaborativo, como metodologías aplicadas a la educación intercultural, al desarrollo de competencias interculturales y a la formación del profesorado para la interculturalidad, son procesos que permiten el trabajo en equipo por la diversidad. Aguado y Mata (2017, pp.22-24) señalan que la formación en educación intercultural debe seguir tres premisas básicas: un enfoque intercultural en educación versus formación en educación intercultural; formación en centros y redes versus formación individual y en grupo; revisión de creencia y prácticas versus entrenamiento en técnicas y metodologías. Para todo ello resultan imprescindibles todos los métodos y técnicas, de tipo cooperativo y colaborativo, que se pueden poner en marcha a través de las metodologías activas. La gestación de estos vínculos debe reafirmar el cuidado y la confianza pedagógica en los estudiantes como legítimos en su diversidad personal y cultural, como seres únicos e irrepetibles. Esto está fuera de toda duda. La participación y la generación de sinergias para aprender a convivir desde la inclusión y la interculturalidad van a posibilitar un cambio positivo, una transformación de la escolaridad en humanidad. 
Porque consignan un aprendizaje significativo, porque no sólo se produce aprendizaje de conocimientos curriculares o académicos, porque permite el desarrollo de habilidades de trabajo en equipo o la valoración de otros puntos de vista a través de la interacción con otros compañeros. También resulta muy apropiado para debatir y cuestionar los conceptos e ideas que se consideran normalmente como conocimientos válidos, contribuyendo así a la construcción conjunta del conocimiento y al aprendizaje por descubrimiento, ayudando a los participantes a ser partícipes auténticos de su aprendizaje, junto con otros compañeros. Todo ello básico a la hora de trabajar la interculturalidad como un vehículo privilegiado para trabajar valores, principios y prácticas escolares de enorme potencialidad para ir generando una educación moderna, una educación inclusiva (Leiva, 2016).

Los objetivos perseguidos en este trabajo se han logrado desde el punto de vista de que se han definido las metodologías activas, y la diferencia que existe cuando al referimos a ellas hablamos de aprendizaje cooperativo y aprendizaje colaborativo. Igualmente se ha perfilado cuál debe ser la principal misión de dichas metodologías a la hora de plantear la puesta en marcha de la formación intercultural del profesorado. Debe quedar claro que este estudio es sólo un punto de partida para posteriores estudios, que aborden con sistematicidad y profundidad en qué medida las metodologías activas forman parte de la formación del profesorado, y de la puesta en marcha de propuestas educativas de carácter intercultural. Las limitaciones de este estudio son las propias de todo estudio teórico, cuya finalidad es describir el estado de la cuestión de un asunto, y describir los rasgos fundamentales del mismo.

\section{Referencias}

Aguado, M. T. (2005). La educación intercultural en la práctica escolar: investigación en el ámbito español. Revista de educación, 7, pp.43-52.

Barkley, E. F., Cross, K. P. \& Major, C. H. (2012). Técnicas de aprendiraje colaborativo: manual para el profesorado universitario (2a. ed.). Madrid: Ediciones Morata.

González-Fernández, N. \& García-González, J. L. (2012). Metodologías participativas para la mejora del aprendizaje en educación superior. Un proyecto innovador con estudiantes de la Facultad de Educación. Revista Iberoamericana para la Investigación y el Desarrollo Educativo, 3(5), pp. 80-93.

Castells, M. (coord.) (2006). La sociedad red: una visión global. Madrid: Alianza Editorial.

Deardorff, D. K. (2009). Intercultural competence model. The Sage Handbook of Intercultural Competence. Thousand Oaks: Sage.

Escarbajal, A (2011). Interculturalidad, mediación y trabajo colaborativo. Madrid: Narcea.

Escarbajal, A. (2010). La escuela inclusiva en una sociedad pluricultural y la importancia del trabajo colaborativo. Enseñanza \& Teaching, 28, pp. 161-179.

Essomba, M. A. (2015). La formación crítica de los profesionales de la educación al servicio de la diversidad cultural y la interculturalidad. En A. Escarbajal Frutos (Ed.), Comunidades interculturales y democráticas: un trabajo colaborativo para una sociedad inclusiva (pp. 127-142). Madrid: Narcea.

Fernández-March, A. (2006). Metodologías activas para la formación de competencias. Educatio siglo XXI, 24, pp. 35-56.

González-Fernández, N. \& García-González, J. L. (2012). Metodologías participativas para la mejora del aprendizaje en educación superior. Un proyecto innovador con estudiantes de la Facultad de Educación. Revista Iberoamericana para la Investigación y el Desarrollo Educativo, 3(5), pp. 80-93.

Jandt, F. (1995). Intercultural Communication. An Introduction. Thousand Oaks: Sage.

Labrador, M. J. \& Andreu, M. A. (Eds.) (2008). Metodologias activas. Grupo de innovación en metodologias activas (GIMA). Valencia: Editorial UPV.

Leiva, J. J. (2011). La educación intercultural en una encrucijada de caminos: reflexiones pedagógicas para la construcción de una escuela intercultural. Espiral.Cuadernos del profesorado, 4(7), pp. 43-56.

Leiva, J. J. (2011a). Principios pedagógicos de la educación para la convivencia intercultural. En J. J. Leiva \& R. Borrero, Interculturalidad y escuela. Perspectivas pedagógicas en la construcción comunitaria de la 
escuela intercultural (pp. 41-66). Barcelona: Octaedro.

Leiva, J. J. (2012). La formación en educación intercultural del profesorado y de la comunidad educativa. Revista Electrónica de Investigación y Docencia (REID). Número monográfico. (Oct.), pp. 8-31.

Leiva, J. J. (2017). La Escuela Intercultural hoy: reflexiones y perspectivas pedagógicas. Revista Complutense de Educación, 28(1), pp. 29-43.

Leiva, J. J., \& Escarbajal, A. (2011). La participación de las familias inmigrantes como fundamento pedagógico en la construcción de la interculturalidad en la escuela. Educatio Siglo XXI: Revista de la Facultad de Educación, 29(2), pp. 389-416.

Malik, B. \& Sánchez, I. (2006). Aprendizaje cooperativo y colaborativo. En T. Aguado, \& M. del Olmo (Eds.) (2006). Educación intercultural perspectivas y propuestas (pp. 201-220). Madrid: Editorial Centros de Estudios Ramón Areces, UNED.

Ortega-Velasco, M. S. (2011). La mirada de la escuela a la interculturalidad: el cimiento de la ciudadanía. Dedica. Revista de Educaçao e Humanidades, 1, pp. 521-532.

Palomero, J. E. (2006). Formación inicial de los profesionales de la educación en Pedagogía intercultural: una asignatura pendiente. El caso de Aragón. Revista Interuniversitaria de Formación del Profesorado, 55 (20, 1), pp. 207-230.

Páramo, M. B., Pérez, R. \& Ruiz, F. J. (2016). Metodologías activas para la formación con tecnologías. En M. J. Gallego-Arrufat \& M. Raposo-Rivas. Formación para la educación con tecnologias (pp. 84-92). Madrid: Difusora Larousse-Ediciones Pirámide.

Peñalva Vélez, A. \& Aguilar, M. J. (2011). Reflexiones sobre la interculturalidad en las aulas. Aportaciones desde la sociología visual. Educación y Diversidad, 5 (1), pp. 73-85.

Peñalva Vélez, A. \& Leiva, J. J. (en prensa). La interculturalidad en el contexto universitario: necesidades en la formación inicial de los futuros profesionales de la educación. Educar.

Peñalva Vélez, A. \& López-Goñi, J. J. (2014). Competencias ciudadanas en alumnado de magisterio: la competencia intercultural personal. Revista Electrónica Interuniversitaria de Formación del Profesorado, 17(2), pp. 139-153.

Peñalva Vélez, A., López-Goñi J .J. \& Barrientos, J. (2017). Habilidades emocionales y profesionalización docente para la educación inclusiva en la sociedad red. Contextos Educativos, 20, pp. 201-215.

Pérez-Torenero, J. M. \& Tejedor, S. (Eds.). (2016). Ideas para aprender a aprender: manual de innovación educativa y tecnología. Barcelona: Editorial UOC.

Prados, M. M., Sánchez, V., Sánchez-Queija, I., Del Rey, R., Pertegal, M. A., Reina, M. D., Ridao, P., Ortega F. J. \& Mora-Merchán, J. (2014). Manual de psicología de la educación para docentes de Educación Infantily Primaria. Madrid: Pirámide.

Pujolàs, P. (2012). Aulas inclusivas y aprendizaje cooperativo. Educatio siglo XXI, 30, pp. 89-112.

Sánchez-Queija, I. \& Pertegal, M. A. (2014). La interacción entre iguales en el aula: el trabajo cooperativo. En M. M. Prados et al. Manual de psicología de la educación para docentes de Educación Infantily Primaria. Madrid: Pirámide.

Santos-Rego, M. A., Cernadas-Ríos, F. X. \& Lorenzo-Moledo, M. M. (2014). La inclusión educativa de la inmigración y la formación intercultural del profesorado. Revista Electrónica Interuniversitaria de Formación del Profesorado, 17(2), pp. 123-137.

Soriano, E. (2005). La interculturalidad como factor de calidad educativa. Madrid: La Muralla S.A.

Trujillo, F. (2010). Promover y fomentar la educación intercultural como un eje fundamental de la formación del profesorado. En R. Flecha, Libro Blanco de la Educación Intercultural, volumen 31(4) (pp. 52-53). Aragón: FETE-UGT.

Zarzar, C. (2016). Instrumentación didáctica por competencias. México: Grupo Editorial Patria. 